\title{
Abstracts
}

\section{ESTIMATION OF POTENTIAL QUALITY-ADJUSTED LIFE YEARS SAVED FROM WEARING A HELIMET ON RIDING A MOTORCYCLE IN TAIWAN}

Cheng-Kang Lee, Jensen Chang, ${ }^{1}$ Cheng-Fen Yu, ${ }^{1}$ Jung-Der Wang ${ }^{2}$ 'NTU, Taipei, Taiwan; ${ }^{2}$ NTU and National Cheng Kung University, Tainan, Taiwan

10.1136/oemed-2011-100382.10

Objectives We have developed method to estimate the costeffectiveness of prevention in occupational and environmental health, which was illustrated with an empirical example of the quantification of the health benefit from wearing helmets among motorcyclists in Taiwan.

Methods We estimated the incidence rate and survival from the registry of head injury. The expected years of life loss was estimated from simulating an age- and sex-matched reference population from the life table of Taiwan. The measurements of the utility value of quality of life were also conducted from a random sample of the registered cohort of head injury, which were multiplied with the survival function to obtain the quality-adjusted life expectancy (QALE) and loss of QALE in quality-adjusted life year (QALY).

Results The cumulative incidence rate between age 45 and 69 was 0.025 , while the average lifetime loss of utility for a case of head injury with and without helmet were 5.8 and 10.7 QALY, respectively. As the relative risks of wearing a full-face helmet and a half-face helmet were 0.31 and 0.73 respectively, compared with no helmet, the expected loss of QALE would be $0.045,0.106$, and 0.268 QALY for each one of them. As the costs of a full-face helmet and a half-face helmet were about 66 , and 33 US dollars, respectively, it appears that wearing a helmet is cost-effective, even if one replaces a new helmet every 3 years.

Conclusions The method is useful to quantify the expected QALY gained from preventive services. 